\title{
Comparison of LAMP, GeneXpert, Mycobacterial Culture, Smear Microscopy, TSPOT.TB, TBAg/PHA Ratio for Diagnosis of Pulmonary Tuberculosis
}

\author{
Yan DENG ${ }^{1}$, Yi-fei DUAN ${ }^{1}$, Shu-pei GAO ${ }^{1}$, Jian-miao WANG ${ }^{1,2 \#}$ \\ ${ }^{1}$ Department of Respiratory and Critical Care Medicine, Tongji Hospital, Tongji Medical College, Huazhong University of \\ Science and Technology, Wuhan 430030, China \\ ${ }^{2}$ Department of Infection Control, Tongji Hospital, Tongji Medical College, Huazhong University of Science and Technology, \\ Wuhan 430030, China
}

(C) Huazhong University of Science and Technology 2021

\begin{abstract}
[Abstract] Objective: To investigate the application value of loop-mediated isothermal amplification (LAMP), GeneXpert, mycobacterial culture, smear microscopy, TSPOT.TB (TSPOT), ratio of TB-specific antigen to phytohemagglutinin (TBAg/PHA ratio) in the detection of mycobacterium tuberculosis in the bronchoalveolar lavage fluid. Methods: A retrospective analysis was performed on the patients who underwent bronchoscopy from December 2018 to November 2019 in Tongji Hospital. The patients with positive tuberculosis culture or positive GeneXpert in bronchoalveolar lavage fluid were selected as the case group, and those without tuberculosis served as the control group. The receiver operating characteristic (ROC) curve was used to evaluate the diagnostic value of LAMP, GeneXpert, culture, smear microscopy, TSPOT, and TBAg/PHA ratio. Results: For the patients with positive cultures as case, the sensitivity of LAMP, GeneXpert, smear microscopy, TSPOT and TBAg/PHA ratio was 73.49\%, 89.16\%, 25.30\%, 80.00\%, 33.85\%, respectively, the specificity was $99.00 \%, 100.00 \%, 99.00 \%, 86.00 \%, 100.00 \%$, respectively, the area under the ROC curve (AUC) was $0.849,0.938,0.633,0.830,0.669$, respectively. For the patients with positive GeneXpert as case, the sensitivity of LAMP, mycobacterial culture, smear microscopy, TSPOT and TBAg/PHA ratio was 73.20\%, 74.23\%, 22.68\%, 68.92\%, 29.73\%, respectively, the specificity was $99.00 \%, 100.00 \%, 99.00 \%, 86.00 \%, 100.00 \%$, respectively, the AUC was $0.853,0.878,0.623,0.775,0.649$, respectively. Conclusion: The sensitivity of GeneXpert was best. The sensitivity and diagnostic value of LAMP were slightly lower than those of GeneXpert, and were similar to tuberculosis culture. The sensitivity of smear microscopy was low. The specificity of TSPOT was low. When TBAg/PHA ratio $>0.2$ was used as a diagnostic index, the specificity was improved, but the sensitivity was low.

Key words: pulmonary tuberculosis; bronchoalveolar lavage fluid; loop-mediated isothermal amplification; GeneXpert; TSPOT
\end{abstract}

Tuberculosis is a chronic infectious disease caused by mycobacterium tuberculosis, which is a major threat to world public health. According to the report of WHO, tuberculosis is one of the ten leading causes of death. Before the outbreak of COVID-19, it had surpassed HIV/AIDS as the leading cause of death from a single infectious disease ${ }^{[1]}$. About a quarter of the world's population has been infected with mycobacterium tuberculosis, with a lifetime risk of $10 \%-15 \%$ of developing tuberculosis. China has the second highest burden of tuberculosis, accounting for about $9 \%$ of the world's annual cases ${ }^{[2]}$. For a long time, the diagnosis

Yan DENG, E-mail: dy102398@163.com

\#Corresponding author, E-mail: wangjianmiao2010@126. com of tuberculosis has been dependent on traditional smear microscopy and culture techniques. The sensitivity of smear microscopy is poor. And it cannot exclude other mycobacteria. The time for culture is too long, which cannot meet the need for early diagnosis.

Rapid and accurate diagnosis is pivotal for the treatment of tuberculosis and the control of tuberculosis transmission ${ }^{[3]}$. In recent years, many researches have been devoted to the development of molecular diagnostic techniques for tuberculosis. The nucleic acid amplification technology (NAAT), a most common kind of molecular diagnostic technology has developed rapidly. GeneXpert is a NAAT method recommended by WHO for tuberculosis diagnosis and rifampicin resistance screening. A half-set of realtime quantitative polymerase chain reaction (PCR) 
amplification technology is used in GeneXpert to automatically extract deoxyribonucleic acid (DNA) and amplify rpoB genes ${ }^{[4]}$. More than $95 \%$ of rifampicin resistant strains had rpoB gene variation, so that drugresistant strains could be identified simultaneously ${ }^{[4]}$. However, GeneXpert requires sophisticated laboratory facilities and severe environmental conditions. Hence it is difficult to develop in the underdeveloped areas in China. Another mycobacterium tuberculosis NAAT technology is called tuberculosis loop-mediated isothermal amplification (LAMP) which is invented by Notomi in $2000^{[5]}$. LAMP is a fast, simple and effective method for DNA amplification. It can accurately amplify several strands of DNA several times in an hour $^{[6]}$. The current tuberculosis LAMP application is based on the amplification of target gene gyrB and IS6110 of mycobacterium tuberculosis complex ${ }^{[7]}$. LAMP is convenience, economy and energy saving. Thus, it can be widely used, especially in countries or regions with lagging resources ${ }^{[8]}$. However, there are few diagnostic researches on LAMP in China.

In recent years, many other new tuberculosis related technologies have also been invented, such as the cellular immune-based mycobacterium tuberculosis interferon release assay (IGRA), of which the most widely used is the TSPOT.TB(TSPOT) test. TSPOT can distinguish mycobacterial infection from BCG vaccination, but cannot distinguish active tuberculosis from latent tuberculosis ${ }^{[9]}$. In order to distinguish active tuberculosis from latent infection, ratio of TB-specific antigen to phytohemagglutinin (TBAg/PHA ratio) was developed based on TSPOT, with high specificity and poor sensitivity ${ }^{[10-11]}$.

Our retrospective study focused on the clinical application of LAMP, GeneXpert, TSPOT, TBAg/PHA ratio, smear microscopy and culture, and compared their sensitivity, specificity and diagnostic efficacy.

\section{MATERIALS AND METHODS}

\subsection{Study Subjects}

Patients admitted to the Department of Respiratory and Critical Care Medicine of Tongji Hospital from December 2018 to November 2019 for bronchoscopy examination with final diagnosis of pulmonary tuberculosis were selected as the case group. Inclusion criteria included: (1) pulmonary lesions; (2) The patients performed bronchoalveolar lavage fluid LAMP, GeneXpert, smear microscopy, mycobacterial culture and blood TSPOT; (3) Mycobacterium tuberculosis culture and/or GeneXpert in bronchoalveolar lavage fluid were positive. In addition, non-tuberculosis patients were selected as the control group. The inclusion criteria included: (1) pulmonary lesions; (2) The patients performed bronchoalveolar lavage fluid LAMP, GeneXpert, smear microscopy, mycobacterial culture and blood TSPOT; (3) Tuberculosis was excluded clinically. The diagnostic criteria for tuberculosis refer to WS 2882017 Tuberculosis Diagnosis published in 2017 ${ }^{[12]}$.

\subsection{Evaluation Index}

The results of LAMP, GeneXpert, smear microscopy, mycobacterial culture, T-SPOT and TBAg/PHA ratio in two groups were compared, and their diagnostic value for pulmonary tuberculosis was evaluated. The evaluation indexes include sensitivity, specificity, positive predictive value, negative predictive value and accuracy. The area under the receiver operating characteristic (ROC) curve (AUC) $>0.5$ and $<0.7$ indicates low diagnostic value, $\geq 0.7$ and $<0.9$ indicates moderate diagnostic value, and $\geq 0.9$ indicates high diagnostic value.

\subsection{Statistical Methods}

GraphPad Prism 7 and SPSS 23.0 software were used for data analysis. Measurement data were represented by mean \pm standard deviation (SD); data in line with normal distribution were analyzed by oneway ANOVA or T-test; otherwise, Mann-Whitney $U$ test was used for analysis, and counting data were analyzed by Chi-square test. The AUC was used to evaluate the diagnostic value. $P<0.05$ was considered statistically significant.

\section{RESULTS}

\subsection{Diagnostic Value of LAMP, GeneXpert, Smear Microscopy, TSPOT and TBAg/PHA Ratio in Pulmonary Tuberculosis Patients with Positive Mycobacterium Tuberculosis Culture as Reference Standard}

There were 83 cases of positive culture, and the median time of mycobacterium culture was 21 (14.533.5) days. There were 100 non-tuberculous patients. There were no significant differences in age and gender between the two groups (table 1). LAMP, GeneXpert, smear microscopy, TSPOT, TBAg/PHA ratio showed the sensitivity of $73.49 \%, 89.16 \%, 25.30 \%, 80.00 \%$, $33.85 \%$, respectively, and the specificity of $99.00 \%$, $100.00 \%$, 99.00\%, 86.00\%, 100.00\%, respectively (table 2). The AUC of LAMP, GeneXpert, smear microscopy, TSPOT and TBAg/PHA ratio was 0.849 , $0.938,0.633,0.830$ and 0.669 , respectively (table 3 , fig. 1).

Table 1 Clinical information of the subjects

\begin{tabular}{lcccc}
\hline Information & $\begin{array}{c}\text { Positive } \\
\text { culture } \\
\text { patients }\end{array}$ & $\begin{array}{c}\text { Non- } \\
\text { tuberculous } \\
\text { patients }\end{array}$ & Statistics & $P$ value \\
\hline$n$ & 83 & 100 & & \\
Age (years) & $48.6 \pm 18.1$ & $52.1 \pm 14.0$ & 3858 & 0.414 \\
Sex & & & 0.094 & 0.759 \\
$\quad$ Male & 50 & 58 & & \\
$\quad$ Female & 33 & 42 & & \\
\hline
\end{tabular}


Table 2 Diagnostic efficacy of LAMP, GeneXpert, smear microscopy, TSPOT, and TBAg/PHA ratio (\%)

\begin{tabular}{lccccc}
\hline Methods & Sensitivity (\%) & Specificity (\%) & PPV (\%) & NPV (\%) & Accuracy (\%) \\
\hline LAMP & 73.49 & 99.00 & 98.39 & 81.82 & 87.43 \\
GeneXpert & 89.16 & 100.00 & 100.00 & 91.74 & 95.08 \\
Smear microscopy & 25.30 & 99.00 & 95.45 & 61.49 & 65.57 \\
TSPOT & 80.00 & 86.00 & 78.79 & 86.87 & 83.64 \\
TBAg/PHA ratio & 33.85 & 100.00 & 100.00 & 69.93 & 73.94 \\
\hline
\end{tabular}

PPV: positive predictive value; NPV: negative predictive value

Table 3 The AUC of LAMP, GeneXpert, smear microscopy, TSPOT, and TBAg/PHA ratio

\begin{tabular}{lccccc}
\hline \multirow{2}{*}{ Methods } & AUC & Standard error & \multirow{2}{*}{$P$} & \multicolumn{2}{c}{$95 \%$ confidence interval } \\
\cline { 4 - 6 } & & & $<0.001$ & 0.779 & Upper limit \\
\hline LAMP & 0.849 & 0.036 & $<0.001$ & 0.891 & 0.919 \\
GeneXpert & 0.938 & 0.024 & 0.004 & 0.543 & 0.986 \\
Smear microscopy & 0.633 & 0.046 & $<0.001$ & 0.761 & 0.899 \\
TSPOT & 0.830 & 0.035 & $<0.001$ & 0.580 & 0.759 \\
TBAg/PHA ratio & 0.669 & 0.046 & & &
\end{tabular}

AUC: area under the curve

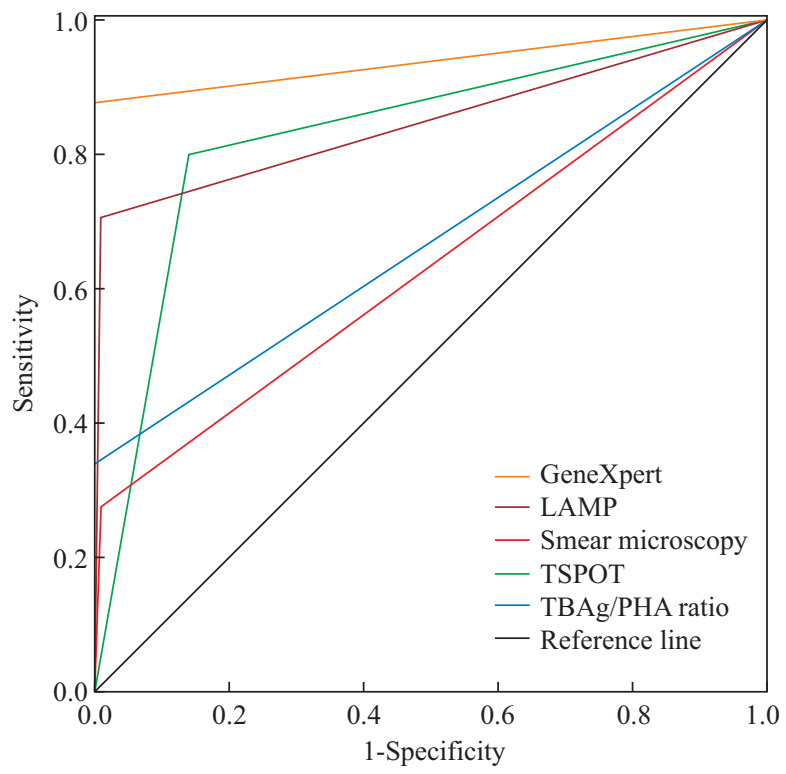

Fig. 1 The AUC of tuberculosis diagnosis by different methods The positive mycobacterium tuberculosis culture patients were regarded as the case group.

2.2 Diagnostic Value of LAMP, Tuberculosis Culture, Smear Microscopy, TSPOT and TBAg/ PHA Ratio in Pulmonary Tuberculosis Patients with Positive GeneXpert as Reference Standard

There were 97 GeneXpert-positive pulmonary tuberculosis patients, including 3 cases of drugresistant tuberculosis, accounting for $3.09 \%$. There were 100 non-tuberculosis patients. There were no significant differences in age and gender between the two groups (table 4). The diagnostic sensitivity of LAMP, culture, smear microscopy, TSPOT, TBAg/PHA ratio was $73.20 \%, 74.23 \%, 22.68 \%, 68.92 \%, 29.73 \%$, respectively (table 4 ), and the specificity was $99.00 \%$, $100.00 \%$, $99.00 \%, 86.00 \%, 100.00 \%$, respectively (table 5). The diagnostic AUC of LAMP, culture, smear microscopy, TSPOT, and TBAg/PHA ratio was 0.853 , $0.878,0.623,0.775$, and 0.649 , respectively (table 6 , fig. 2).

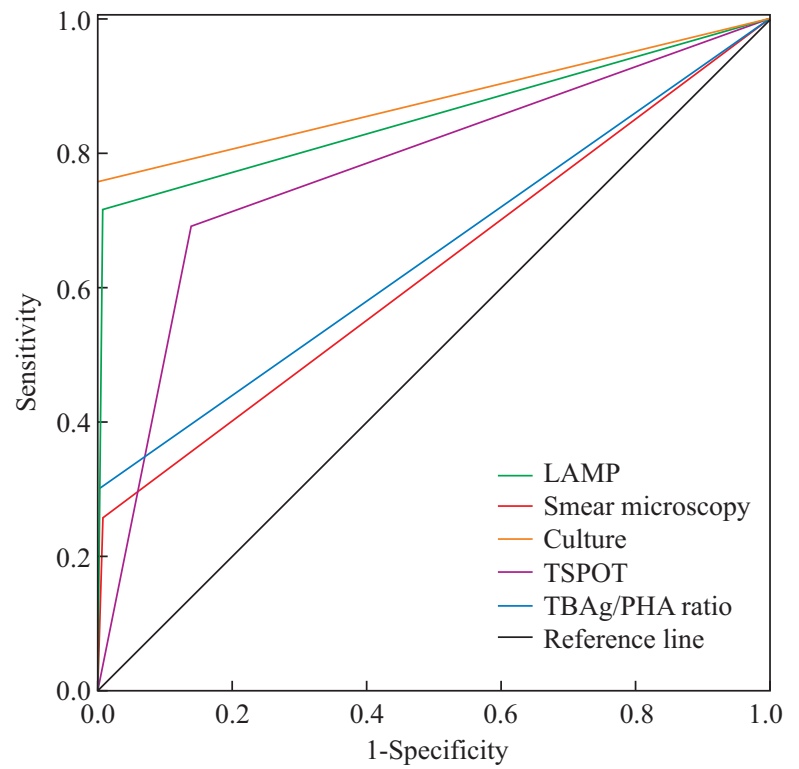

Fig. 2 The AUC of tuberculosis diagnosis by different methods The positive GeneXpert patients were regarded as the case group.

Table 4 Clinical information of the subjects

\begin{tabular}{lcccc}
\hline Information & $\begin{array}{c}\text { Positive } \\
\text { GeneXpert } \\
\text { patients }\end{array}$ & $\begin{array}{c}\text { Non- } \\
\text { tuberculous } \\
\text { patients }\end{array}$ & Statistics & $P$ value \\
\hline$n$ & 97 & 100 & & \\
Age (years) & $50.2 \pm 16.6$ & $52.1 \pm 14.0$ & 4746 & 0.796 \\
Sex & & & 0.012 & 0.914 \\
$\quad$ Male & 57 & 58 & & \\
Female & 40 & 42 & & \\
\hline
\end{tabular}


Table 5 Diagnostic efficacy of LAMP, culture, smear microscopy, TSPOT, and TBAg/PHA ratio (\%)

\begin{tabular}{lccccc}
\hline Methods & Sensitivity (\%) & Specificity (\%) & PPV (\%) & NPV (\%) & Accuracy (\%) \\
\hline LAMP & 73.20 & 99.00 & 98.61 & 79.20 & 86.29 \\
Culture & 74.23 & 100.00 & 100.00 & 80.00 & 87.31 \\
Smear microscopy & 22.68 & 99.00 & 95.65 & 56.90 & 61.42 \\
TSPOT & 68.92 & 86.00 & 78.46 & 78.90 & 78.74 \\
TBAg/PHA ratio & 29.73 & 100.00 & 100.00 & 65.79 & 70.11 \\
\hline
\end{tabular}

PPV: positive predictive value; NPV: negative predictive value

Table 6 The AUC of LAMP, culture, smear microscopy, TSPOT, and TBAg/PHA ratio

\begin{tabular}{lccccc}
\hline \multirow{2}{*}{ Methods } & AUC & Standard error & \multirow{2}{*}{$P$} & \multicolumn{2}{c}{$95 \%$ confidence interval } \\
\cline { 4 - 6 } & & & & Lower limit & Upper limit \\
\hline LAMP & 0.853 & 0.033 & $<0.001$ & 0.788 & 0.918 \\
Culture & 0.878 & 0.031 & 0.001 & 0.818 & 0.939 \\
Smear microscopy & 0.623 & 0.044 & $<0.001$ & 0.537 & 0.710 \\
TSPOT & 0.775 & 0.038 & 0.001 & 0.700 & 0.849 \\
TBAg/PHA ratio & 0.649 & 0.044 & & 0.563 & 0.735 \\
\hline
\end{tabular}

AUC: area under the curve

\section{DISCUSSION}

Pulmonary lesion size, sputum volume, specimen retention method, immunity state, drug use, and the characteristics of mycobacterium tuberculosis may all affect the clinical diagnosis of pulmonary tuberculosis. Hence the negative rate of sputum is relatively high ${ }^{[13]}$. Fiberoptic bronchoscopy lavage can be performed directly in the site of bronchial lesions to obtain a relatively high concentration of mycobacterium tuberculosis. It can reduce specimen contamination, improve the detection rate, and is applicable to the detection of patients without expectoration ${ }^{[14]}$. There are various tuberculosis-related tests for bronchoscopy lavage fluid as we discussed before. Different tests have different advantages and disadvantages. It is important to choose an economical and effective method, especially in resource-poor areas.

Smear microscopy and mycobacterium culture are the traditional methods. The smear microscopy is simple and quick, but its sensitivity and repeatability are poor. Smear quality, staining time and microscopic examination experience all affect the positive rate, and tuberculosis bacilli are difficult to be detected if the patient is not in the bacteria elimination stage ${ }^{[15]}$. In addition, acid-fast staining positive specimen may also be non-mycobacterium tuberculosis and mycobacterium leprae ${ }^{[15]}$. In this study, the specificity of the smear microscopy of bronchoscopy lavage fluid was high in the positive culture group the same as that of the positive GeneXpert group, but the sensitivity was very low, only $20 \%-30 \%$, which was similar to the previous research $^{[16]}$. Mycobacterium tuberculosis culture is the gold standard in the diagnosis of tuberculosis at present. A study showed that in the sputum smearnegative suspected tuberculosis patients, the sensitivity of bronchoalveolar lavage mycobacterium tuberculosis culture was $57.1 \%{ }^{[17]}$. Our study showed that the sensitivity of bronchoalveolar lavage mycobacterium tuberculosis culture was $74.23 \%$ in GeneXpert-positive patients. The biggest drawback of tuberculosis culture is the long culture cycle, which can't meet the needs of clinical diagnosis. The median time for mycobacterium tuberculosis culture in our study was 21 days.

The development of nucleic acid amplification technology has opened up a new area for tuberculosis diagnosis. In recent years, the World Health Organization has recommended molecular diagnostic methods such as GeneXpert to promote the diagnosis of tuberculosis and drug-resistant tuberculosis ${ }^{[18]}$. A result of a multicenter study using mycobacterium tuberculosis culture as the diagnostic criteria suggested that the sensitivity and specificity of GeneXpert were $92.2 \%$ and $99.2 \%$ respectively ${ }^{[19]}$. Another study on the diagnostic efficacy in some countries and regions has shown that GeneXpert, as a preliminary screening method for suspected mycobacterium tuberculosis infection, had a total sensitivity of $88 \%$ and specificity of $98 \%{ }^{[20]}$. Studies have suggested that the sensitivity and specificity of GeneXpert may be decreased in smearnegative patients and HIV patients ${ }^{[2]}$. The sensitivity of GeneXpert in smear-negative patients was about $68 \%$, but the specificity was more than $97 \%{ }^{[21]}$. In different studies, the sensitivity of GeneXpert in the detection of mycobacterium tuberculosis in bronchoalveolar lavage fluid is different, which varies from $81 \%$ to $97 \%$. In addition, GeneXpert can be used to confirm culturenegative tuberculosis ${ }^{[22,23]}$. In our study, the sensitivity of GeneXpert was $89.16 \%$ when positive tuberculosis culture was taken as the standard, which was consistent to the results of previous studies.

Studies about LAMP in developing countries 
showed that its sensitivity in sputum smear-positive tuberculosis patients was $97.7 \%$, and that was $48.8 \%$ in smear-negative tuberculosis patients, and the specificity was $99.0 \%{ }^{[24]}$. Another study suggested that the overall sensitivity and specificity of LAMP for tuberculosis diagnosis were $99 \%$ and $94 \%$ respectively ${ }^{[25]}$. A recent meta-analysis showed that its detection sensitivity and specificity of sputum specimens were similar to those of GeneXpert ${ }^{[26]}$. However, there are few studies about the LAMP method in bronchoalveolar lavage fluid to detect mycobacterium tuberculosis. A research in China comparing LAMP with tuberculosis culture included a total of 51 clinically diagnosed pulmonary tuberculosis patients, and found that the positive rate of LAMP method in the bronchoalveolar lavage fluid of pulmonary tuberculosis patients was $54.9 \%$, significantly higher than $5.9 \%$ for acid-fast smear and $35.3 \%$ for mycobacterial culture ${ }^{[27]}$. In our study, the sensitivity and specificity of LAMP in the detection of mycobacterium tuberculosis in bronchoalveolar lavage fluid were $73 \%$ and $99 \%$ respectively. Its diagnostic value was also similar to that of tuberculosis culture method, slightly lower than that of GeneXpert. LAMP has low requirements for equipment and environment. Besides, it showed high stability, even those technicians who have no experience in molecular biology experiments can operate it skillfully. It is fast, simple, economical and efficient.

TSPOT is a test for tuberculosis using the peripheral blood. A previous study shows that, with the healthy subjects as a comparison, the sensitivity and specificity for the diagnosis of active tuberculosis were $91.0 \%$ and $75.2 \%$ respectively ${ }^{[11]}$. The application of TBAg/PHA ratio is aimed at distinguishing active tuberculosis and latent infections. A previous study showed the AUC to active tuberculosis diagnosis for $\mathrm{TBAg} / \mathrm{PHA}$ ratio was 0.881 , which is significantly greater than that for TSPOT ${ }^{[1]}$. In our study, when the threshold value of TBAg/PHA ratio was set as 0.2 in reference to the above researches, its specificity was $100 \%$, but its sensitivity was only about $30 \%$, which also meant its diagnostic value was low.

In conclusion, our study found that the sensitivity and diagnostic value of smear microscopy were poor. The traditional tuberculosis culture takes a long time. GeneXpert has the best sensitivity, diagnostic value, and can complete drug resistance tests simultaneously. But it's expensive and requires advanced laboratory equipment and is not yet widely available. LAMP is economical and fast, and its sensitivity and specificity are slightly lower than those of GeneXpert, which is similar to those of mycobacterial culture. LAMP is worth promoting, especially in settings where GeneXpert is not available and where drug-resistance is not of concern. The sensitivity of blood TSPOT in the positive culture tuberculosis patients was higher than that of positive GeneXpert tuberculosis patients. In general, the specificity of blood TSPOT was low. When TBAg/PHA ratio $>0.2$ was used as a diagnostic index, the specificity was improved, but the sensitivity was low. The combination of all approaches can help to improve the detection of tuberculosis, but it also increases the costs. In clinical practice, detection techniques can be selected or utilized comprehensively according to their advantages and the local medical conditions.

\section{Conflict of Interest Statement}

All authors declare no conflict of interest.

\section{REFERENCES}

1 World Health Organization(2019). Global tuberculosis report 2019. 2019, WHO, Geneva. Available from: https://apps.who.int/iris/bitstream/hand le/10665/329368/9789241565714-eng.pdf. [Accessed 30 June 2021].

2 World Health Organization(2018). Global tuberculosis report 2018. 2018, WHO, Geneva. Available from: https://apps.who.int/iris/bitstream/hand le/10665/274453/9789241565646-eng.pdf. [Accessed 30 June 2021].

3 Pang Y, Du J, Qin ZZ, et al. An overview on tuberculosisspecific hospitals in China in 2009: results of a national survey. Eur Respir J, 2016,47(5):1584-1587

4 Lawn SD, Nicol MP. Xpert® MTB/RIF assay: development, evaluation and implementation of a new rapid molecular diagnostic for tuberculosis and rifampicin resistance. Future Microbiol, 2011,6(9):10671082

5 Notomi T, Okayama H, Masubuchi H, et al. Loopmediated isothermal amplification of DNA. Nucleic Acids Res, 2000,28(12):E63

6 Rudeeaneksin J, Bunchoo S, Srisungngam S, et al. Rapid identification of Mycobacterium tuberculosis in BACTEC MGIT960 cultures by in-house loopmedicated isothermal amplification. Jpn J Infect Dis, 2012,65(4):306-311

$7 \mathrm{Ou}$ X, Li Q, Xia H, et al. Diagnostic accuracy of the PURE-LAMP test for pulmonary tuberculosis at the county-level laboratory in China. PLoS One, 2014,9(5):e94544

8 Neonakis IK, Spandidos DA, Petinaki E. Use of loopmediated isothermal amplification of DNA for the rapid detection of Mycobacterium tuberculosis in clinical specimens. Eur J Clin Microbiol Infect Dis, 2011,30(8):937-942

9 Wang S, Zhang WH. New progress on diagnosis of tuberculosis. J Microbes Infect, 2016,11(3):188-192

10 Wang F, Hou HY, Wu SJ, et al. Using the TBAg/PHA ratio in the T-SPOT( $\left({ }^{\circledR}\right)$.TB assay to distinguish TB disease from LTBI in an endemic area. Int J Tuberc Lung Dis, 2016,20(4):487-493

11 Wang T, Tan YJ, Wu SJ, et al. The ratio of tuberculosisspecific antigen to phytohemagglutinin in T-SPOT assay in the diagnosis of active tuberculosis. Chin J Tuberc Respir Dis, 2019,42(4):262-267

12 National Health and Family Planning Commission 
of the People's Republic of China. Diagnosis for pulmonary tuberculosis. The health industry standard of the People's Republic of China, WS 288-2017, 2017, Available from: http://www.nhc.gov.cn/wjw/ s9491/201712/a452586fd21d4018b0ebc00b89c06254. shtml. [Accessed 30 June 2021].

13 Song QZ, Li H, Shao H, et al. MicmRNA-365 in macrophages regulates mycobacterium-induced active pulmonary tuberculosis via inteleukin-6. Int J Clin Exp Med, 2015,8(9):15 458-15 465

14 Bodal VK, Bal MS, Bhagat S, et al. Fluorescent microscopy and Ziehl-Neelson staining of brochoalveolar lavage, bronchial washings, brochoscpic brushing and post bronchoscopic sputum along with cytological examination in cases of suspectec tuberculosis. Indial J Pathol Microbiol, 2015,58(4):443-447

15 Steingart KR, Henry M, Ng V, et al. Fluorescence versus conventional sputum smear microscopy for tuberculosis: a systematic review. Lancet Infect Dis, 2006,6:570e81

$16 \mathrm{Xu}$ P, Tang P, Song HF, et al. The incremental value of bronchoalveolar lavage for the diagnosis of pulmonary tuberculosis in a high-burden urban setting. J Infect, 2019,79(1):24-29

17 Liu X, Hou XF, Gao L, et al. Indicators for prediction of Mycobacterium tuberculosis positivity detected with bronchoalveolar lavage fluid. Infect Dis Poverty, 2018,7(1):22

18 World Health Organization(2013). Automated realtime nucleic acid amplification technology for rapid and simultaneous detection of tuberculosis and rifampicin resistance: Xpert MTB/RIF assay for the diagnosis of pulmonary and extrapulmonary $\mathrm{TB}$ in adults and children: policy Update. 2013, WHO, Geneva. Available from: https:/apps.who.int/iris/ bitstream/handle/10665/112472/9789241506335_eng. pdf? sequence $1 / 41$. [Accessed 30 June 2021].

19 Boehme CC, Nabeta P, Hillemann D, et al. Rapid molecular detection of tuberculosis and rifampin resistance. N Engl J Med, 2010,363(11):1005-1015

20 Steingart KR, Sohn H, Schiller I, et al. Xpert ${ }^{\circledR}$ MTB/ RIF assay for pulmonary tuberculosis and rifampicin resistance in adults. Cochrane Database Syst Rev, 2013, (1):CD009593

21 Boehme CC, Nicol MP, Nabeta P, et al. Feasibility, diagnostic accuracy, and effectiveness of decentralised use of the Xpert MTB/RIF test for diagnosis of tuberculosis and multidrug resistance: a multicentre implementation study. Lancet, 2011,377(9776):14951505

22 Lee HY, Seong MW, Park SS, et al. Diagnostic accuracy of Xpert ${ }^{\circledR}$ MTB/RIF on bronchoscopy specimens in patients with suspected pulmonary tuberculosi. Int $\mathrm{J}$ Tuberc Lung Dis, 2013,17(7):917-921

23 Pan X, Yang S, Deighton MA, et al. A Comprehensive Evaluation of Xpert MTB/RIF Assay with Bronchoalveolar Lavage Fluid as a Single Test or Combined with Conventional Assays for Diagnosis of Pulmonary Tuberculosis in China: A Two-Center Prospective Study. Front Microbiol, 2018,9:444

24 Boehme CC, Nabeta P, Henostroza G, et al. Operational feasibility of using loop-mediated isothermal amplification for diagnosis of pulmonary tuberculosis in microscopy centers of developing countries. J Clin Microbiol, 2007,45(6):1936-1940

25 Bojang AL, Mendy FS, Tientcheu LD, et al. Comparison of TB-LAMP, GeneXpert MTB/RIF and culture for diagnosis of pulmonary tuberculosis in The Gambia. J Infect, 2016,72(3):332-337

26 Shete PB, Farr K, Strnad L, et al. Diagnostic accuracy of TB-LAMP for pulmonary tuberculosis: a systematic review and meta-analysis. BMC Infect Dis, 2019,19(1):268

27 Wang H, Liu QX, Chen L, et al. Evaluation of LAMP method in detecting BALF specimens from patients clinically diagnosed with pulmonary tuberculosis. Int $\mathbf{J}$ Lab Med, 2019,40(7):783-786

(Received Nov. 26, 2020; accepted Mar. 23, 2021) 